\title{
MSW-28, a Full-flavor Crisp Watermelon Line with High Lycopene and Medium Brix
}

\author{
Angela R. Davis ${ }^{1}$ \\ U.S. Department of Agriculture, Agricultural Research Service, South \\ Central Agricultural Research Laboratory, P.O. Box 159, Highway 3 West, \\ Lane, OK 74555
}

\section{Stephen R. King}

Vegetable \& Fruit Improvement Center, Department of Horticultural Sciences, Texas A\&M University, College Station, TX 77843

Additional index words. Citrullus lanatus, taste test, lower sugar, firm flesh, heirloom

The Agricultural Research Service of the U.S. Department of Agriculture announces the release of MSW-28, a watermelon [Citrullus lanatus var. lanatus (Thunb.) Matsum. \& Nakai)] line that exhibits medium sugar content (brix) and full flavor of heirloom varieties combined with the lycopene content and crisp texture of modern cultivars. Most heirloom cultivars of watermelon have a full flavor but typically lower sugar content compared with modern cultivars. Many people report a preference for heirloom varieties, citing flavor as the primary reason. Unfortunately, heirloom varieties often have low yield, poor disease resistance, and inferior flesh texture and quality. Increasing fruit sugar content has been a priority of many breeding programs, and the sugar content of modern watermelon cultivars is considerably higher than their heirloom counterparts. It is estimated that there are 20.8 million people in the United States with diabetes (Centers for Disease Control and Prevention, 2005). Watermelon is considered a high-glycemic food (Foster-Powell et al., 2002) but a good source of lycopene (Perkins-Veazie et al.,

\footnotetext{
Received for publication 9 May 2007. Accepted for publication 24 June 2007.

We thank Amy Helms and Bill Anderson for providing valuable technical support and Dr. James Shrefler for providing conventional watermelon for taste testing.

Mention of trade names or commercial products in this article is solely for the purpose of providing specific information and does not imply recommendation or endorsement by the U.S. Department of Agriculture. All programs and services of the U.S. Department of Agriculture are offered on a nondiscriminatory basis without regard to race, color, national origin, religion, sex, age, marital status, or handicap. The article cited was prepared by a USDA employee as part of his or her official duties. Copyright protection under U.S. copyright law is not available for such works. Accordingly, there is no copyright to transfer. The fact that the private publication in which the article appears is itself copyrighted does not affect the material of the U.S. Government, which can be freely reproduced by the public.

${ }^{1}$ To whom reprint requests should be addressed; e-mail adavis-usda@lane-ag.org.
}

2006), vitamin A, and potassium (U.S. Department of Agriculture, Agricultural Research Service, 2006). The goal of this research was to develop a watermelon variety adapted to modern farming practices that has a firm rind; firm, crisp, full-flavored high lycopene flesh with small funiculus; and a moderate brix for consumers interested in fully flavored, lower sugar fruit.

\section{Origin}

Efforts that led to the development of MSW-28 began in 2001, 1 year after our breeding plots and neighboring fields were planted with multiple commercial varieties and several Plant Introduction lines. Seeds from an escape, open-pollinated $C$. lanatus var. lanatus exhibiting low brix, very firm intense yellow flesh, and firm rind, discovered in a field near our breeding plots in Lane, OK, in the Fall of 2001, were used to develop MSW-28. We developed this line to exhibit firm rind, firm, full-flavored high lycopene flesh, and lower brix than commercially available cultivars as a specialty market fruit that could cater to consumers interested in moderate carbohydrate diets. Controlled pollinations of seed from this plant were initiated in the winter of 2003 in a greenhouse. Fruit from nine progeny plants were analyzed for brix and flesh pigmentation (based on a visual color rating). The three fruit with the highest red pigmentation and lowest brix were selected and grown in a greenhouse with controlled pollinations. Fruit were selected for high pigmentation and low brix with controlled self-pollinations for four generations followed by one open-pollination in an isolated field at which point the fruit phenotype appeared to be fixed. However, we collected seed from the fruit with the best overall appearance and performed another self-pollination in a greenhouse during the fall of 2006 to produce $\mathrm{F}_{6}$-derived MSW-28 seed.

\section{Plant Characteristics}

Approximately $50 \mathrm{~F}_{6}$ plants were evaluated during the summer of 2006 in Lane, OK.
Transplants were planted 3 feet apart in one row of a nonreplicated field. They demonstrated uniform fruit characteristics and produced $\approx 2$ fruit per plant. MSW-28 is a midseason monoecious plant. Typical fieldgrown MSW-28 plants produce small $(5 \mathrm{~kg})$ elongated fruit with a medium to dark green rind and dark green stripes similar to 'Allsweet' (Fig. 1) and a firm rind, comparable to 'Black Diamond'. It has firm red flesh, an average brix content of 9.0, and an average lycopene content of $66 \mu \mathrm{g} \cdot \mathrm{g}^{-1}$ for ripe fruit. MSW-28 demonstrated lower brix and equivalent or improved lycopene content when compared with 'Dixielee' (10.2 and 68.1, respectively) and 'Black Diamond' (9.7 and 33.8, respectively) grown in our fields (Perkins-Veazie et al., 2006). Brix was tested using a digital refractometer (Atago model PR 100, Gardiner, NY) and lycopene was measured as described by Davis et al. (2003).

Taste tests were performed according to Collins et al. (2006). MSW-28 was sweetened with sucrose to match the brix content of a commercially available open-pollinated variety ('Allsweet') with a brix of 12.0. This variety was chosen because of its similar color and texture to MSW-28. Sucrose was added to both varieties to match the brix in all samples and to allow for the consistency change resulting from the added sugar. The final brix for these samples was 14.0. The following were rated: the amount of watermelon flavor, sweetness, overall flavor, texture, and whether the taste tester liked or disliked the sample. There was no significant difference in acceptability between varieties for any of the categories tested (Fig. 2). Because there was no significant difference in the acceptability of the brix-matched fruits, we can conclude that when sweetness is not considered, MSW-28 is as acceptable as a fully sweet commercial variety. A similar test comparing unsweetened MSW28 (brix of 9.0) with fully sweet ('Allsweet') watermelon (brix of 12.0) indicated only a slight preference by most taste testers for the watermelon flavor, sweetness, and overall
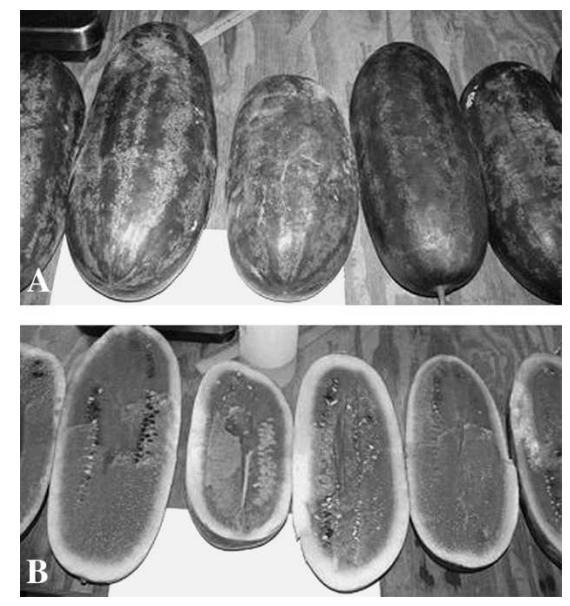

Fig. 1. Ripe MSW-28 fruit showing typical fruit shape and rind pattern (A) and typical rind thickness and red flesh color (B). 
Sucrose Added

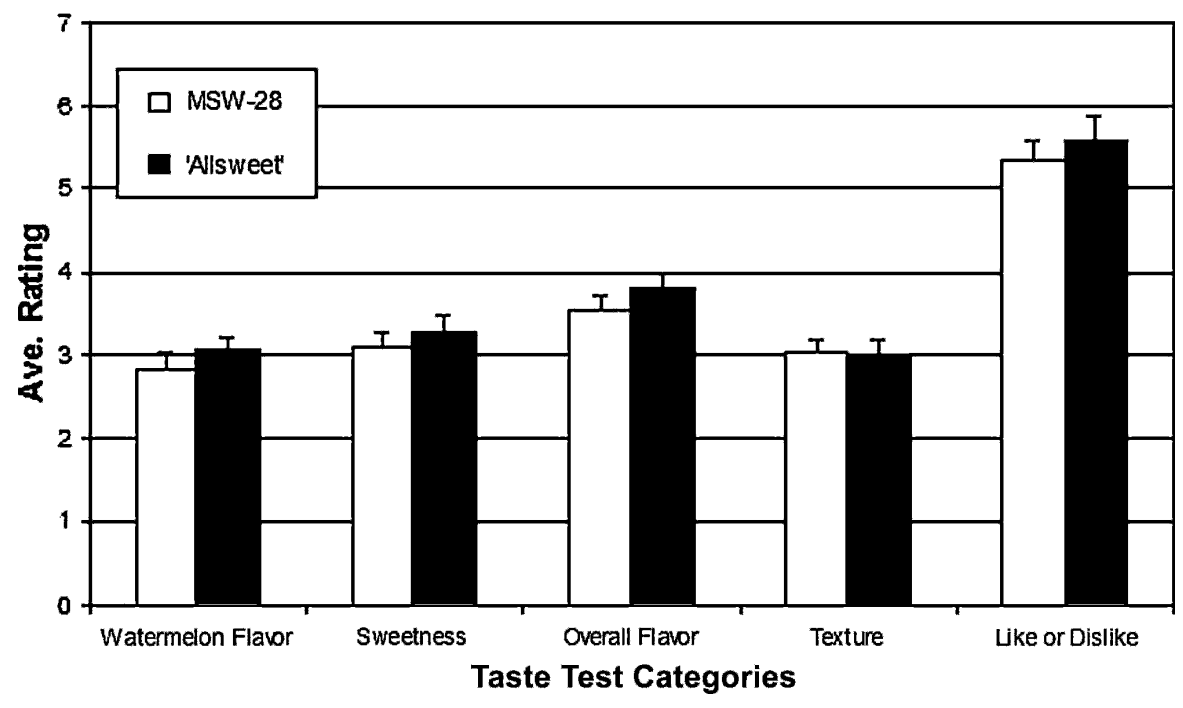

Fig. 2. Taste test results from 15 test subjects. Each bar is a representation of the average rating for each subject tasting two blind-labeled samples for each variety. Sucrose was added to both varieties to match brix and consistency change resulting from the added sugar. Samples were rated on a one to four, one to five, or a one to seven scale, depending on the trait being tested, with one being least desirable. Maximum value for "watermelon flavor" was 4; for "sweetness" 4; for "overall flavor" 5; for "texture" 4; and for "like or dislike" 7. Error bars represent the sE from the mean within each melon type and within each category.

No Sucrose Added

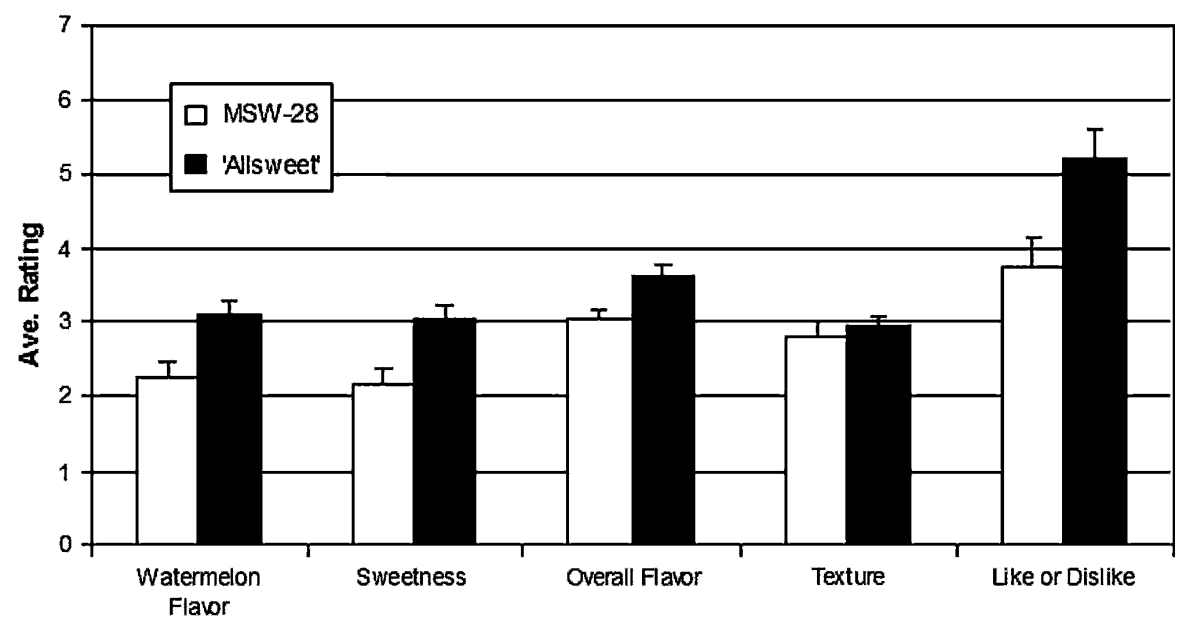

Taste Test Categories

Fig. 3. Taste test results from 15 test subjects. Each bar is a representation of the average rating for each subject tasting two blind-labeled samples for each variety. No sucrose was added to either of the varieties. Samples were rated on a one to four, one to five, or a one to seven scale, depending on the trait being tested, with one being least desirable. Maximum value for "watermelon flavor" was 4 ; for "sweetness" 4; for "overall flavor" 5; for "texture" 4; and for "like or dislike" 7. Error bars represent the SE from the mean within each melon type and within each category.

flavor, and they indicated that they liked the fully sweet cultivar more (Fig. 3). However, of the 15 taste testers, there was a subset

( $27 \%$ of testers) that consistently preferred the medium brix samples or ranked them the same as the control fruit. These data suggest that medium brix watermelon lines may represent a unique consumer product.

MSW-28 is a specialty market fruit that could cater to consumers interested in moderate carbohydrate diets containing substantial levels of vitamins and phytonutrients from fresh fruits and vegetables. MSW-28 will be useful for producers growing for niche and specialty markets, home gardeners, and plant breeders who develop germplasm for niche and specialty markets.

\section{Availability}

Small samples of MSW-28 seed are available to research personnel and plant breeders who make a written request to Dr. Angela R. Davis, South Central Agricultural Research Laboratory, P.O. Box 159, Lane, OK, 74555 (e-mail: adavis-usda@lane-ag.org). MSW28 seed will be submitted to the National Plant Germplasm System where it will be available for research purposes, including the development and commercialization of new cultivars. It is requested that appropriate recognition of the source be given when this germplasm contributes to research or development of a new breeding line or cultivar.

\section{Literature Cited}

Centers for Disease Control and Prevention National diabetes fact sheet: General information and national estimates on diabetes in the United States. 2005. U.S. Department of Health and Human Services, Centers for Disease Control and Prevention, Atlanta, GA. 18 Apr. 2007. <http://www.diabetes.org/uedocuments/ NationalDiabetesFactSheetRev.pdf/>.

Collins, J.K., A.R. Davis, A. Adams, N. Mannes, and P. Perkins-Veazie. 2006. Consumer acceptability of low sugar watermelon sweetened with non-calorie sweetener by a Native American community. Int. J. Food Sci. Nutr. 57: $363-368$.

Davis, A.R., W.W. Fish, and P. Perkins-Veazie. 2003. A rapid hexane-free method for analyzing lycopene content in watermelon. J. Food Sci. 68:328-332.

Foster-Powell, K., S.H. Holdt, and J.C. BrandMiller. 2002. International table of glycemic index and glycemic load values: 2002. Amer. J. Clin. Nutr. 76:5-56.

Perkins-Veazie, P., J.K. Collins, A.R. Davis, and W. Roberts. 2006. Carotenoid content of watermelon cultivars. J. Agr. Food Chem. 54:25932597.

U.S. Department of Agriculture, Agricultural Research Service. 2006. USDA National Nutrient Database for Standard Reference, Release 19. Nutrient Data Laboratory Home Page. U.S. Dept. Agr., Washington, DC. 23 Apr. 2007. <http://www.ars.usda.gov/ba/bhnrc/ndl/> 